Les ANNALES POLONICI MATHEMATICI constituent une continuation des ANNALES DE LA SOCIÉTE POLONAISE DE MATHÉMATIQUE (vol. I-XXV) fondées en 1921 par Stanisław Zaremba.

Les ANNALES POLONICI MATHEMATICI publient, en langues des congrès internationaux, des travaux consacrés à l'Analyse Mathématique, la Géométrie et la Théorie des Nombres. Chaque volume parait en $\mathbf{3}$ fascicules.

Les manuscrits dactylographiés sont à expédier à l'adresse: AT POLONICI MATHEMATICI

KRAKOW (Pologne), ul. Solskiego 30.

Toute la correspondance concernant l'échange et l'administration est

$$
\text { à expédier à l'adresse: }
$$

ANNALES POLONICI MATHEMATICI

WARSZAWA 10 (Pologne), ul. Sniádeckich 8.

Le prix de ce fascicule est. $2 \$$.

Les ANNALES sont à obtenir par l'intermédiaire de ARS POLONA

WarszaWa 5 (Pologne), Krakowskie Przedmieście 7.

PRINTED IN POLAND

\section{Sur un pseudogroupe infini et un problème d'équivalence}

par H. Pidek-Łopuszańska et W. ŚLebodziński (Wrocław)

On sait bien que le groupe orthogonal $O_{n}$ n'est pas involutif, d'où il s'ensuit qu'il n'existe pas de pseudogroupe infini de Lie dont le groupe de structure soit $O_{n}$ et que, par conséquent, l'espace euclidien n'admet pas de déformations au sens d'E. Cartan. Il n'en est pas de même du groupe linéaire des matrices de la forme

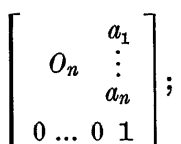

ce groupe étant involutif, il peut jouer le rôle de groupe de structure d'un pseudogroupe infini $G$ de transformations de $n+1$ variables indépendantes; nous désignerons ce pseudogroupe par $G$. Ses transformations, qui dépendent de $n$ fonctions arbitraires d'une variable, représentent les déformations du premier ordre de l'espace de Galilée. Au $\mathrm{n}^{0} 1$ de cet article nous établissons les équations de définition et les équations de structure de pseudogroupe $G$. Aux nos 2,3 et 4 nous utilisons ces équations pour déterminer les invariants du système d'équations

$$
\frac{d x^{h}}{d t}=u^{h}\left(x^{1}, x^{2}, \ldots, x^{n}, t\right)
$$

par rapport aux transformations $d u$ pseudogroupe $G$, autrement dit nous trourons les conditions d'équivalence de deux systèmes d'équations de la forme (*). Nous montrons enfin ( $\left.n^{\circ} 5\right)$ qu'aux équations $(*)$ on peut associer d'une manière invariante une connexion affine, ce qui permet de géométriser ce système d'équations et le problème de l'équivalence.

1. Soit $\Gamma$ le groupe de transformations

$$
\bar{x}_{h}=\sum_{r} a_{h r} x_{r}+a_{h} t+b_{h}, \quad \llbracket \bar{t}=t
$$

de $n+1$ variables $\left.x_{h}{ }^{1}\right), t, a_{h r}$ désignant les éléments d'une matrice orthogonale $O_{n}$ de déterminant égal à +1 et $a_{h}, b_{h}$ des paramètres

(1) Les indices latins parcourent les valeurs $1,2, \ldots, n$. 
arbitraires. Les transformations de $\Gamma$, qui est appelé groupe de Galilée, dépendent ainsi de $n(n+3) / 2$ paramètres arbitraires. L'espace à $n+1$ dimensions de coordonnées $x_{h}, t$, basé sur le groupe $\Gamma$, sera appelé espace de Galilée. Nous dirons que dans l'espace de Galilée on a défini un vecteur, si dans chaque système de coordonnées déduit du système $x_{h}, t$ au moyen d'une transformation du groupe $T$ on a donné une suite de composantes $V_{0}, V_{1}, \ldots, V_{n}$ et que ces composantes se transforment d'après les formules

$$
\bar{V}_{h}=\sum_{r} a_{h r} V_{r}+a_{h} V_{0}, \quad \bar{V}_{0}=V_{0}
$$

si l'on passe des coordonnées $x_{h}, t$ aux coordonnées $\bar{x}_{h}, \bar{t}$. Un repère $R_{n+1}$ composé de $n+1$ vecteurs $I^{a}(a=0,1, \ldots, n)$ de composantes $V_{0}^{a}, V_{1}^{a}, \ldots, V_{n}^{a}$ sera dit galiléen, si ces composantes satisfont aux relations

$$
V_{h}^{0}=0, \quad V_{0}^{h}=0, \quad \sum_{r} V_{r}^{h} V_{r}^{i}=\delta^{h i} .
$$

Un repère galiléen se transforme en repère galiléen, si l'on change les coordonnées au moyen d'une transformation du groupe de Galilée.

Pour obtenir les équations de structure du groupe $\Gamma$ posons

$$
\omega_{h}=\sum_{r} \alpha_{h r} d x_{r}+\alpha_{h} d t
$$

en désignant par $a_{h r}$ des paramètres satisfaisant aux relations

$$
\sum_{r} \alpha_{h r} \alpha_{i r}=\sum_{r} \alpha_{h r} \alpha_{r i}=\delta_{h i}, \quad\left|\alpha_{h i}\right|=+1
$$

et par $\alpha_{h}$ des paramètres arbitraires. En résolvant les équations (1.1) par rapport aux différentielles $d x_{r}$ et en tenant compte des relations (1.2) on trouve

$$
\text { (1.3) } \quad d x_{r}=\sum_{i} \alpha_{i r} \omega_{i}-\sum_{i} \alpha_{i r} \alpha_{i} d t .
$$

Différentions maintenant extérieurement les équations (1.1) et remplaçons ensuite les différentielles $d x_{r}$ par les expressions (1.3); on obtient ainsi les relations

$$
d \omega_{h}+\sum_{i}\left[\omega_{h i} \omega_{i}\right]+\left[\tau_{h} d t\right]=0,
$$

où l'on a posé

$$
\omega_{h i} \doteq \sum_{r} \alpha_{h r} d \alpha_{i r}, \quad \tau_{h}=-d \alpha_{h}+\sum_{i, r} \alpha_{i r} \alpha_{i} d \alpha_{h r}
$$

remarquons qu'en vertu de (1.2) il vient

$$
\omega_{h i}+\omega_{i h}=0 \text {. }
$$

En différentiant les formes $\omega_{h i}$ et $\tau_{h}$ on obtient les équations

$$
d \omega_{h i}+\sum_{r}\left[\omega_{h r} \omega_{r i}\right]=0, \quad d \tau_{h}+\sum_{r}\left[\omega_{h r} \tau_{r}\right]=0
$$

qu'il est facile de vérifier.

Les relations (1.4) et (1.7) sont les équations de structure du groupe qui est le prolongement holoédrique du groupe $\Gamma$ aux transformations des paramètres $\alpha_{h i}$ et $\alpha_{h}$; les équations de définition de ce groupe peuvent être écrites comme il suit:

$$
\bar{t}=t, \quad \bar{\omega}_{h}=\omega_{h}, \quad \bar{\omega}_{h i}=\omega_{h i}, \quad \bar{\tau}_{h}=\tau_{h},
$$

$\bar{\omega}_{h}, \bar{\omega}_{h i}, \bar{\tau}_{h}$ désignant respectivement ce que deviennent les formes $\omega_{h}, \omega_{h i}$ et $\tau_{h}$, si l'on y remplace les variables $x_{h}, t, \alpha_{h i}$ et $\alpha_{h} \operatorname{par} \bar{x}_{h}, \bar{t}, \bar{a}_{h i}$ et $\bar{\alpha}_{h}$. Il est facile de voir, eu égard aux équations de structure, que le système d'équations (1.8) est complètement intégrable.

Si l'on restreint le système (1.8) aux équations

$$
\bar{t}=t, \quad \bar{\omega}_{h}=\omega_{h},
$$

on obtient un système de Pfaff dont les solutions définissent les déformations du premier ordre de l'espace de Galilée ( $\left.{ }^{2}\right)$. Ce système est en involution relativement aux variables indépendantes $x_{h}, t, a_{h i}$ et $a_{h}$ de sa solution générale. Pour s'en convaincre il faut le fermer en différentiant les équations (1.9); si l'on tient compte des formules (1.4) et des relations (1.9), on obtient les équations

$$
\sum_{i}\left[\left(\bar{\omega}_{h i}-\omega_{h i}\right) \omega\right]+\left[\left(\bar{\tau}_{h}-\tau_{h}\right) d t\right]=0
$$

qui montrent bien l'involutivité du système (1.9). On voit donc que le groupe de déformations de l'espace de Galilée est un groupe infini dont le groupe de structure est le groupe des matrices

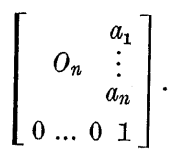

Nous désignerons ce groupe infini par $G$. Il est facile de vérifier que les équations finies de ce groupe sont données par les formules

$$
\bar{x}_{h}=\sum_{r} A_{h r}(t) x_{r}+A_{h}(t), \quad \bar{t}=t,
$$

(2) E. Cartan, Sur le problème général de la déformation, C. R. Congrès de Stras bourg 1920, p. 317-406. 
où $A_{h r}(t)$ sont des fonctions de la variable $t$ assujetties aux relations

$$
\sum_{r} A_{h r} A_{r i}=\sum_{r} A_{r h} A_{r i}=\delta_{h i}
$$

et $A_{h}(t)$ des fonctions arbitraires. Les transformations du groupe $G$ dépendent done de $n(n+1) / 2$ fonctions arbitraires d'une variable. Remarquons que les équations (1.10), qui représentent les déformations de l'espace de Galilée à $n+1$ dimensions, peuvent être aussi conçues comme les équations du mouvement d'un corps rigide dans l'espace euclidien à $n$ dimensions de coordonnées orthogonales $x_{n}$.

Si dans l'espace de Galilée on se donne un objet géométrique, par exemple une famille de courbes ou de surfaces définie au moyen d'un système d'équations différentielles, on peut se poser le problème de trouver les invariants de cet objet par rapport au groupe de déformations $G$ et de résoudre le problème de l'équivalence de deux objets de même genre; aux invariants ainsi définis nous donnerons le nom d'invariants de déformation. Dans les numéros suivants nous allons étudier un des problèmes de cette nature.

2. Supposons que l'on se donne dans l'espace de Galilée deux systèmes d'équations différentielles

$$
\begin{aligned}
\frac{d x_{h}}{d t} & =u_{h}\left(t, x_{1}, x_{2}, \ldots, x_{n}\right) \\
\frac{d \bar{x}_{h}}{d \bar{t}} & =\bar{u}_{h}\left(\bar{t}_{1}, \bar{x}_{1}, \bar{x}_{2}, \ldots, \bar{x}_{n}\right)
\end{aligned}
$$

dont chacun représente le mouvement d'un milieu continu dans l'espace euclidien à $n$ dimensions, $t$ représentant le temps; nous supposons que $u_{h}$ et $\bar{u}_{h}$ sont des fonctions analytiques dans un ouvert connexe de l'espace de Galilée. Nous nous proposons de trouver les conditions d'équivalence de ces systèmes pas rapport au groupe de déformation $G$. Ce problème peut aussi être énoncé de la manière suivante: établir les conditions pour que le mouvement (2.1) du milieu continu se manifeste comme mouvement (2.2) pour un observateur lié à un corps rigide qui se meut dans l'espace euclidien suivant les équations (1.10).

Pour résoudre ce problème posons

$$
\theta_{h}=\sum_{r} q_{h r}\left(d x_{r}-u_{r} d t\right), \quad \bar{\theta}_{h}=\sum_{r} \bar{q}_{h r}\left(d \bar{x}_{r}-\bar{u}_{r} d t\right),
$$

où $q_{h r}$ et $\bar{q}_{h r}$ désignent des paramètres arbitraires; pour que les systèmes (2.1) et (2.2) soient équivalents par rapport au groupe $G$, il faut et il suffit que les solutions du système d'équations de Pfaff (1.9) entrainent les égalités $\bar{\theta}_{h}=\theta_{h}$. Le problème proposé se ramène donc à l'étude du système d'équations

$$
\bar{t}=t, \quad \bar{\omega}_{h}=\omega_{h}, \quad \bar{\theta}_{h}=\theta_{h} .
$$

Or en substituant les expressions (1.3) dans la première des formules (2.3) il vient

$$
\theta_{h}=\sum_{j, r} q_{h r} \alpha_{j r} \omega_{j}-\left(\sum_{r} q_{h r} u_{r}+\sum_{j, r} \alpha_{j r} \alpha_{j} q_{h r}\right) d t .
$$

Nous choisirons les paramètres arbitraires de manière que les coefficients de $\omega_{j}$ et de $d t$ dans la dernière formule prennent respectivement les valeurs numériques $\delta_{j h}$ et 0 ; on doit donc poser

$$
\sum_{r} q_{h r} \alpha_{j r}=\delta_{h j}, \quad \sum_{r} q_{h r} u_{r}+\sum_{j, r} \alpha_{j r} \alpha_{j} q_{h r}=0 .
$$

En multipliant la première de ces égalités par $\alpha_{j r}$ et en sommant par rapport à $j$ on obtient, en vertu des relations (1.2), l'égalité $q_{h i}=\alpha_{h i}$. Si l'on remplace $q_{h r}$ par $\alpha_{h r}$ dans la seconde des relations (2.5), il viendra $\alpha_{h}+\sum_{r} \alpha_{h r} u_{r}=0$. On voit ainsi que les relations (2.5) sont équivalentes aux suivantes

$$
q_{h i}=\alpha_{h i}, \quad \alpha_{h}=-\sum_{r} \alpha_{h r} u_{r} .
$$

Si l'on en tient compte, les formes $\omega_{h}$ et $\theta_{h}$ définies par les formules (1.1) et (2.3) deviendront identiques

$$
\omega_{h}=\theta_{h}=\sum_{r} \alpha_{h r}\left(d x_{r}-u_{r} d t\right)
$$

et le système des équations (2.4) se réduira aux relations

$$
\bar{t}=t, \quad \bar{\omega}_{h}=\omega_{h} .
$$

Nous en déduirons de nouvelles équations en différentiant extérieurement. les équations (2.8). Or, de la formule (2.7) on obtient ainsi

$$
d \omega_{h}=\sum_{r}\left[d \alpha_{h r}\left(d x_{r}-u_{r} d t\right)\right]-\sum_{s} \alpha_{h s} \frac{\partial u_{s}}{\partial x_{r}}\left[d x_{r} d t\right]
$$

ce qui peut aussi s'écrire comme il suit:

$$
d \omega_{h}=-\sum_{r}\left[\left(d x_{r}-u_{r} d t\right)\left(d \alpha_{h r}+\sum_{r} a_{h s} \frac{\partial u_{s}}{\partial x_{r}} d t\right)\right] .
$$

Si nous y remplaçons la différence $d x_{r}-u_{r} d t$ par son expression tirée de l'équation (2.7), il viendra

$$
d \omega_{h}=-\sum_{i, r} \alpha_{i r}\left[\omega_{i} d \alpha_{h r}\right]-\sum_{i, r_{s} s} \alpha_{i r} \alpha_{h s} \frac{\partial u_{s}}{\partial x_{r}}\left[\omega_{i} d t\right]
$$


Posons maintenant

$$
\xi_{r s}=\frac{1}{2}\left(\frac{\partial u_{r}}{\partial x_{s}}-\frac{\partial u_{s}}{\partial x_{r}}\right), \quad \eta_{r s}=\frac{1}{2}\left(\frac{\partial u_{r}}{\partial x_{s}}+\frac{\partial u_{s}}{\partial x_{r}}\right)
$$

et remarquons que ces grandeurs jouissent des propriétés suivantes

$$
\xi_{r s}+\xi_{s r}=0, \quad \eta_{r s}=\eta_{s r} .
$$

Si l'on porte l'expression $\partial u_{s} / \partial x_{r}=\eta_{r s}-\xi_{r s}$ dans l'équation (2.9), on obtient la formule

$$
d \omega_{h}=-\sum_{i, r} \alpha_{i r}\left[\omega_{i} d \alpha_{h r}\right]-\sum_{i, r, s} \alpha_{i r} \alpha_{h s} \xi_{r s}\left[\omega_{i} d t\right]-\sum_{i, r, s} \alpha_{i r} \alpha_{h s} \eta_{r s}\left[\omega_{i} d t\right],
$$

qui peut étre écrite de la manière suivante

$$
d \omega_{h}=\sum_{i, r}\left[\left(-\alpha_{h r} d a_{i r}+\sum_{s} a_{i r} \alpha_{h s} \xi_{r s} d t\right) \omega_{i}\right]-\sum_{i, r, s} \alpha_{h s} \alpha_{i r} \eta_{r s}\left[\omega_{i} d t\right] .
$$

Si l'on y introduit encore les notations

$$
\begin{gathered}
\omega_{h i}=\sum_{r} \alpha_{h r} d \alpha_{i r}+\sum_{r, s} \alpha_{h r} \alpha_{i s} \xi_{r s} d t \\
\Lambda_{h}=-\sum_{i, r, s} \alpha_{h s} \alpha_{i r} \eta_{r s}\left[\omega_{i} d t\right]
\end{gathered}
$$

on obtient enfin la formule

$$
d \omega_{h}+\sum_{i}\left[\omega_{h i} \omega_{i}\right]=\Lambda_{h}
$$

Remarquons que des formules $(2.10),(2.12)$ et (1.2) résulte la relation

$$
\omega_{h i}+\omega_{i h}=0 \text {. }
$$

Observons maintenant que les équations $(2.8)$ du problème de l'équivalence entraînent les relations $d \bar{\omega}_{h}=d \omega_{h}$ qui peuvent s'écrire, en vertu des formules (2.14), comme il suit

$$
\sum_{i}\left[\left(\bar{\omega}_{h i}-\omega_{h i}\right) \omega_{i}\right]=\bar{\Lambda}_{h}-\Lambda_{h}
$$

Si l'on y tient compte de l'expression (2.13), on en déduit l'équation

$$
\sum_{i}\left[\left(\bar{\omega}_{h i}-\omega_{h i}\right) d t\right]=-\sum_{i, r, s}\left(\bar{\alpha}_{h s} \bar{\alpha}_{i r} \bar{\eta}_{r s}-\alpha_{h s} \alpha_{i r} \eta_{r s}\right)\left[\omega_{i} d t\right]
$$

Les formes $\omega_{i}$ et dt étant indépendantes, on doit avoir des relations de la forme suivante

$$
\bar{\omega}_{h i}-\omega_{h i}=\sum_{j} \gamma_{h i j} \omega_{j}+\gamma_{h i} d t
$$

où les coefficients satisfont, d'après les équations (2.15), aux relations

$$
\gamma_{h i j}+\gamma_{i h j}=0, \quad \gamma_{h i}+\gamma_{i h}=0 .
$$

En portant les expressions (2.18) dans les relations (2.16) il viendra

$$
\sum_{i, j} \gamma_{h i j}\left[\omega_{j} \omega_{i}\right]+\sum_{i} \gamma_{h i}\left[d t \omega_{i}\right]=-\sum_{i, r, s}\left(\bar{\alpha}_{h s} \bar{\alpha}_{i r} \bar{\eta}_{r s}-a_{h s} \alpha_{i r} \eta_{r s}\right)\left[\omega_{i} d t\right]
$$

Cette relation entre les formes indépendantes $\omega_{i}$ et $d t$ devant être identiquement satisfaite, il en résulte

$$
\gamma_{h i j}=\gamma_{h j i}
$$

$$
\gamma_{h i}=\sum_{r, s}\left(\bar{\alpha}_{h s} \bar{\alpha}_{i r} \bar{\eta}_{r s}-\alpha_{h s} \alpha_{i r} \eta_{r s}\right)
$$

Remarquons que l'égalité (2.20) et la première des relations (2.19) entraînent la nullité des coefficients $\gamma_{h i j}$; il en est de même pour les coefficients $\gamma_{h i}$, car le premier membre de l'égalité (2.21) est symétrique gauche par rapport aux indices $h, i$ d'après la seconde des relations (2.18) et le second est symétrique par rapport aux mêmes indices en vertu des relations (2.11). Ceci établi, l'équation (2.17) devient

$$
\bar{\omega}_{h i}=\omega_{h i}
$$

de cette équation et de la relation (2.16) résulte qu'on doit aroir

$$
\overline{\Lambda_{h}}=\Lambda_{h} \text {. }
$$

Si l'on différentie la forme $\omega_{h i}$ définie par la formule (2.12), on obtient après un calcul facile la relation

où l'on a posé

$$
d \omega_{h i}+\sum_{r}\left[\omega_{h r} \omega_{r i}\right]=\Lambda_{h i}
$$

$\Omega_{h i j}$ étant défini par la formule

$$
\mathfrak{S}_{h i j}=\sum_{r, s, t} \alpha_{h r} \alpha_{i s} \alpha_{j t} \frac{\partial \zeta_{r s}}{\partial x_{t}} .
$$

Il est facile de vérifier qu'en vertu des relations (2.10) les coefficients $\Omega_{h i j}$ jouissent des propriétés suivantes

$$
\Omega_{h i j}+\Omega_{i h j}=0, \quad \Omega_{h i j}+\Omega_{i j h}+\Omega_{j h i}=0 .
$$


En différentiant l'équation (2.22) et en tenant compte de la formule (2.24) on trouve ensuite que la relation

$$
\bar{\Lambda}_{h i}=\Lambda_{h i}
$$

est aussi une conséquence des équations (2.8).

3. Nous démontrerons maintenant le théorème suivant.

Tнв́ов亡̀me. Le problème de l'équivalence des systèmes différentiels (2.1) et (2.2) par rapport au groupe $G$ équivaut à celui de l'équivalence des deux systèmes

$$
\left\{t, \omega_{h}\right\} \quad \text { et } \quad\left\{\bar{t}, \bar{\omega}_{h}\right\} \text {. }
$$

Nous avons déjà montré que l'équivalence des systèmes (2.1) et (2.2) entraîne les égalités

$$
\bar{t}=t, \quad \bar{\omega}_{h}=\omega_{h}
$$

d'une façon plus précise nous pouvons dire: s'il existe une transformation (1.10) du groupe $G$ qui transforme le système (2.1) en système (2.2), elle peut être prolongée aux variables $\alpha_{h i}$ de telle manière que les équations (3.1) soient satisfaites. Nous démontrerons maintenant la réciproque: s'il existe, entre les variables $t, x_{h}, \alpha_{h i}$ d'une part et les variables $\bar{t}, \bar{x}_{h}, \bar{\alpha}_{h i}$ de l'autre, des relations qui entraînent les équations (3.1), on peut tirer de ces relations des formules de la forme (1.10) qui transforment les systèmes (2.1) et (2.2) l'un dans l'autre.

En effet, il résulte des égalités $\bar{\omega}_{h}=\omega_{h}, \bar{t}=t$ que les relations en question doivent contenir des équations de la forme

$$
\bar{x}_{r}=\bar{x}_{r}\left(t, x_{1}, \ldots, x_{n}\right) .
$$

Si l'on substitue ces expressions dans les équations $\bar{\omega}_{h}=\omega_{h}$, $\omega_{h}$ étant défini par la formule (2.7), on obtient

$$
\sum_{r, s} \bar{a}_{h r}\left(\frac{\partial \bar{x}_{r}}{\partial x_{s}} d x_{s}+\frac{\partial \bar{x}_{r}}{\partial t} d t-\bar{u}_{r} d t\right)=\sum_{r} a_{h r}\left(d x_{r}-u_{r} d t\right)
$$

On en déduit les équations

$$
\sum_{r} \bar{a}_{h r} \frac{\partial \bar{x}_{r}}{\partial x_{s}}=\alpha_{h s}, \quad \sum_{r} \bar{\alpha}_{h r}\left(\frac{\partial \bar{x}_{r}}{\partial t}-\bar{u}_{r}\right)=-\sum_{r} \alpha_{h r} u_{r} .
$$

Les paramètres $\alpha_{h r}$ étant les coefficients d'une substitution orthogonale on déđuit de la première des équations (3.3) la relation

$$
\frac{\partial \bar{x}_{r}}{\partial x_{s}}=\sum_{h} \bar{\alpha}_{h r} a_{h s}
$$

\section{Posons}

(3.4)

par suite il vient

$$
a_{r s}=\sum_{h} \bar{\alpha}_{h r} \alpha_{h s}
$$

$$
\frac{\partial \bar{x}_{r}}{\partial x_{s}}=a_{r s}
$$

Les fonctions $a_{r s}$ étant les coefficients d'une substitution orthogonale il en résulte qu'on doit avoir $\partial^{2} \bar{x}_{r} / \partial x_{8} \partial x_{u}=0$ et que, par conséquent, les formules (3.2) doivent avoir la forme

$$
\bar{x}_{r}=\sum_{s} a_{r s}(t) x_{s}+a_{r}(t)
$$

La transformation cherchée appartient donc au groupe $G$. Il faut maintenant montrer que les expressions (3.6) transforment les équations (2.1) en les équations (2.2). Or, de la deuxième des relations (3.3) résulte l'égalité suivante

$$
\frac{\partial \bar{x}_{i}}{\partial t}-\bar{u}_{i}=-\sum_{h} \bar{\alpha}_{h i} \alpha_{h r} u_{r}
$$

ce qui peut, d'après la formule (3.4), être écrit comme il suit

$$
\frac{\partial \bar{x}_{i}}{\partial t}-\bar{u}_{i}=-\sum_{r} a_{i r} u_{r}
$$

Enfin, si l'on tient compte de l'équation (3.5), on obtient

$$
\frac{\partial \bar{x}_{i}}{\partial t}-\bar{u}_{i}=-\sum_{r} \frac{\partial \bar{x}_{i}}{\partial x_{r}} u_{r}
$$

Cela étant, supposons que dans 1'expression $d \bar{x}_{i}-\bar{u}_{i} d t$ on ait remplacé les variables $\bar{x}_{i}$ par leurs valeurs tirées de la formule (3.5); on aura

$$
d \bar{x}_{i}-\bar{u}_{i} d t=\sum \frac{\partial \bar{x}_{i}}{\partial x_{r}} d x_{r}+\left(\frac{\partial \bar{x}_{i}}{\partial t}-\bar{u}_{i}\right) d t
$$

Eu égard à la relation (3.7) on en déduit l'égalité suivante

$$
d \bar{x}_{i}-\bar{u}_{i} d t=\sum_{r} \frac{\partial \bar{x}_{i}}{\partial x_{r}}\left(d x_{r}-u_{r} d t\right)
$$

ce qui établit le Théorème.

4. Le problème de l'équivalence des systèmes $(2.1)$ et $(2.2)$ a été ramené à l'étude du système d'équations (3.1). Or nous avons vu que ces équations entraînent les relations (2.23) et (2.28) qui peuvent 
être ramenées, en vertu des formules (2.13) et (2.26), aux relations suivantes

$$
\begin{gathered}
\sum_{r s} \bar{\alpha}_{h s} \bar{\alpha}_{i r} \bar{\eta}_{r s}=\sum_{r, s} \alpha_{h s} \alpha_{i r} \eta_{r s} \\
\sum_{r, s, t} \bar{\alpha}_{h r} \bar{\alpha}_{i s} \bar{\alpha}_{j t} \cdot \frac{\partial \bar{\xi}_{r s}}{\partial \bar{x}}=\sum_{r, s, t} \alpha_{h r} \alpha_{i s} \alpha_{j t} \frac{\partial \xi_{r s}}{\partial x_{t}} .
\end{gathered}
$$

Si l'on désigne respectivement par $\alpha$ et $\eta$ les matrices $\left[\alpha_{i j}\right]$ et $\left[\eta_{i j}\right]$, les relations (4.1) peuvent être écrites sous la forme

d'où l'on déduit les égalités

$$
\bar{\alpha} \bar{\eta} \bar{\alpha}^{-1}=\alpha \eta \alpha^{-1}
$$

$$
\begin{aligned}
\operatorname{det} \bar{\eta} & =\operatorname{det} \eta \\
\operatorname{tr} \bar{\eta} & =\operatorname{tr} \eta \\
\operatorname{tr} \bar{\eta}^{2} & =\operatorname{tr} \eta^{2} \\
\operatorname{tr} \bar{\eta}^{n-1} & =\operatorname{tr} \eta^{n-1}
\end{aligned}
$$

Nous nous bornerons dans la suite au cas, assez général, où les premiers membres des équations (4.3) sont des fonctions indépendantes de $\bar{x}^{1}, \ldots, \bar{x}^{n}$, les seconds membres jouissant d'une propriété analogue. On peut alors dire que, si le système (3.1) admet une solution, celle-ci peut être déterminée par les équations (4.3). Pour que le problème d'équivalence admette une solution, il faut donc et il suffit que les équations (4.3) entraînent les égalités (3.1).

Pour simplifier l'écriture mettons les équations (4.3) sous la forme

$$
\bar{F}_{i}=F_{i} .
$$

On en déduit les relations $d \bar{F}_{i}=d F_{i}$ ou

$$
\bar{F}_{i j} d \bar{x}^{j}+\bar{F}_{i 0} d t=F_{i j} d x_{j}+F_{i 0} d t
$$

où l'on a posé $F_{i j}=\partial F_{i} / \partial x_{j}, F_{i 0}=\partial F_{i} / \partial t$ et $\bar{F}_{i j}=\partial \bar{F}_{i} / \partial \bar{x}_{j}, \bar{F}_{i 0}=\partial \bar{F}_{i} / \partial t$. Si l'on y remplace les différentielles $d x^{i}$ et $d x^{i}$ par leurs expressions tirées des formules (2.7) et des formules analogues pour les formes $\bar{\omega}_{h}$, on obtient

$$
\sum_{h, j} \bar{F}_{i j}\left(\bar{\alpha}_{h j} \bar{\omega}_{h}+\bar{u}_{j} d t\right)+\bar{F}_{i 0} d t=\sum_{h, j} \bar{F}_{i j}\left(\alpha_{h j} \omega_{h}+u_{j} d t\right)+F_{i 0} d t .
$$

Les équations (4.4) devant entraîner les relations (3.1), les égalités ci-dessus conduisent aux équations suivantes:

$$
\sum_{h, j}\left(\bar{\alpha}_{h j} \bar{F}_{i j}-\alpha_{h j} F_{i j}\right) \omega_{h}+\left(\sum_{j} \bar{F}_{i j} \bar{u}_{j}+\bar{F}_{i 0}-\sum_{j} F_{i j} u_{j}-F_{i 0}\right) d t=0
$$

Les formes $\omega_{h}$ et $d t$ étant indépendantes, il en résulte

$$
\begin{aligned}
\sum_{j} \bar{\alpha}_{h j} \bar{F}_{i j} & =\sum_{j} \alpha_{h j} F_{i j}, \\
\sum_{j} \bar{F}_{i j} \bar{u}_{j}+\bar{F}_{i 0} & =\sum_{j} F_{i j} u_{j}+F_{i 0} .
\end{aligned}
$$

Les relations (4.6) nous disent que les vecteurs $\bar{F}_{1 j}, \bar{F}_{2 j}, \ldots, \bar{F}_{n j}$ s'obtiennent à partir des $n$ vecteurs $F_{1 j}, F_{2 j}, \ldots, F_{n j}$ au moyen d'une substitution orthogonale; on doit donc avoir

$$
\sum_{k} \bar{F}_{i k} \bar{F}_{j k}=\sum_{k} F_{i k} F_{j k}
$$

Nous voyons ainsi que, si le système différentiel (3.1) admet une solution, celle-ci doit satisfaire aux relations finies (4.4), (4.7) et (4.8). Nous établirons maintenant la résiproque: si ces relations sont compatibles, elles ont pour conséquence les égalités (3.1). En effet, les relations (4.4) entraînent les égalités (4.5) qui peuvent, en vertu des relations (4.7), s'écrire comme il suit

$$
\sum_{j} \bar{F}_{i j}\left(d \bar{x}_{j}-\bar{u}_{j} d t\right)=\sum_{j} F_{i j}\left(d x_{j}-u_{j} d t\right)
$$

Les fonctions $F_{i}$ étant par hypothèse indépendantes, on a $\left|F_{i j}\right| \neq 0$; il en est de même de $\left|\bar{F}_{i j}\right|$. On voit done que les équations (4.4), (4.6) et (4.8) entraînent des relations de la forme (3.1).

Nous avons ainsi démontré que la compatibilité des équations (4.4), (4.6) et (4.8) est une condition nécessaire et suffisante pour que les systèmes $(2.1)$ et $(2.2)$ soient équivalents par rapport au pseudogroupe $G$. Ce théorème n'est valable que si les équations (4.4) permettent d'exprimer les variables $\bar{x}_{k}$ au moyen de $x_{k}$ et $t$.

5. Nous montrerons maintenant qu'au système des équations (2.1) donné dans un ouvert $U$ de l'espace $E^{n+1}$ aux coordonnées $x_{1}, x_{2}, \ldots, x_{n}, t$ on peut associer d'une manière invariante une connexion linéaire.

Nous dirons dans ce but que les grandeurs $A_{\boldsymbol{x}}(x, t)(x=0,1, \ldots, n)\left({ }^{3}\right)$ définissent un vecteur $A$ attaché au point $M(x, t) \in U$, si elles se transforment comme il suit

$$
\bar{A}_{0}=A_{0}, \quad \bar{A}_{h}=\sum_{r} \frac{\partial \bar{x}_{h}}{\partial x_{r}} A_{r}+\frac{\partial \bar{x}_{h}}{\partial t} A_{0}
$$

$\left(^{s}\right)$ Dans tout ce qui suit les indices grecs parcourent les valeurs $0,1, \ldots, n$ et les indices latins les valeurs $1,2, \ldots, n$. 
pour un changement de coordonnées défini au moyen des formules suivantes

$$
\bar{x}_{h}=\bar{x}_{h}\left(x_{1}, x_{2}, \ldots, x_{n}, t\right), \quad \bar{t}=t, \quad \frac{\partial\left(\bar{x}_{1}, \bar{x}_{2}, \ldots, \bar{x}_{n}\right)}{\partial\left(x_{1}, x_{2}, \ldots, x_{n}\right)} \neq 0
$$

Pour définir la connexion demandée associons au point $M$ un espace galiléen $R^{n+1}$ composé de $n+1$ vecteurs $I_{a}\left(\mathrm{n}^{\circ} 1\right)$ et admettons que le mouvement infinitésimal de $R^{n+1}$ soit déterminé par les formules

$$
d M=\sum_{a} \omega_{a} I_{\alpha}, \quad d I_{\alpha}=\sum_{\beta} \omega_{\alpha \beta} I_{\beta}
$$

$$
\begin{gathered}
\omega_{0}=d t, \quad \omega_{h}=\sum_{r} \alpha_{h r}\left(d x_{r}-u_{r} d t\right), \\
\omega_{a 0}=\omega_{0 a}=0, \quad \omega_{h i}=\sum_{r} \alpha_{h r} d \alpha_{i r}+\sum_{r, s} \alpha_{h r} \alpha_{i s} \xi_{r s} d t ;
\end{gathered}
$$

tous les symboles qui y figurent ont la même signification que dans les $n^{\text {os }} 1$ et 2 . On aura par suite $\omega_{h i}+\omega_{i h}=0$.

En différentiant extérieurement les équations (5.2) on obtient les équations de structure de la connexion

$$
d \omega_{a}+\sum_{\varrho}\left[\omega_{a \varrho} \omega_{\varrho}\right]=\Lambda_{\alpha}, \quad d \omega_{a \beta}+\sum_{\varrho} \omega_{a \varrho} \omega_{\beta \varrho}=\Lambda_{a \beta},
$$

où l'on doit poser $\Lambda_{0}=\Lambda_{a 0}=\Lambda_{0 a}=0, \Lambda_{h}$ et $\Lambda_{h i}$ étant définis par les formules (2.13) et (2.25). Les formes $\Lambda_{\alpha}$ et $\Lambda_{\alpha \beta}$ représentent respectivement la torsion et la courbure de la connexion. Nous désignerons par $H_{n+1}$ la connexion ainsi définie, dont le groupe de structure est le groupe $\Gamma$ de Galilée ( $\mathrm{n}^{0}$ 1).

Pour que la torsion de la connexion $H_{n+1}$ soit nulle, il faut et il suffit, d'après la formule (2.13), que l'on ait $\eta_{r s}=0$, c'est-à-dire que la déformation pure d'un liquide, qui se meut d'après les équations (2.1), soit nulle. On sait que dans ce cas le milieu continu se meut comme un corps rigide. Nous pouvons donc énoncer le théorème suivant.

THÉORÈME. Pour que le milien continu dont le mouvement est defini par les équations (2.1) se meuve comme un corps rigide, il faut et il suffit que la torsion de la connexion $H_{n+1}$ soit nulle.

Remarquons encore que, si la déformation pure $\eta_{h i} d u$ milieu continu est nulle, on a $\partial u_{h} / \partial x_{i}+\partial u_{i} / \partial x_{h}=0$ en vertu de la seconde des formules (2.10) et, par suite, les fonctions $u_{h}$ dans les équations (2.1) ont la forme suivante

$$
u_{h}=\sum_{i} p_{h i}(t) x_{i}+p_{h}(t), \quad p_{h i}+p_{i h}=0 .
$$

Les coefficients $\xi_{h i}$, définis par les formules $(2.10)$ ne dépendant pas des variables $x_{j}$, la courbure de la connexion est nulle d'après les formules (2.25) et $(2.26)$. On a donc:

THÉORÈME. Si la torsion de la connexion $H_{n+1}$ est nulle, il en est de même de la courbure.

Revenons maintenant aux équations (2.1) et (2.2); d'après ce qui précède à chacun de ces systèmes d'équations on peut associer d'une manière invariante une connexion linéaire dont le groupe de structure est le groupe de Galilée. Pour que ces connexions, que nous désignerons respectivement par $H_{n+1}$ et $\bar{H}_{n+1}$, soiènt équivalentes il faut et il suffit que l'on ait

$$
\bar{\omega}_{\alpha}=\omega_{\alpha}, \quad \bar{\omega}_{a \beta}=\omega_{\alpha \beta} .
$$

Or, il résulte des raisonnements faits aux n ${ }^{\text {os }} 2-4$ que ces conditions entraînent l'équivalence des deux mouvements (2.1) et (2.4) par rapport au groupe de déformations $G$ et, inversement, l'équivalence des systèmes (2.1) et (2.2) par rapport au groupe $G$ entraîne celle des connexions $H_{n+1}$ et $\bar{H}_{n+1}$.
Regu par la Rédaction le 16.12.1959 